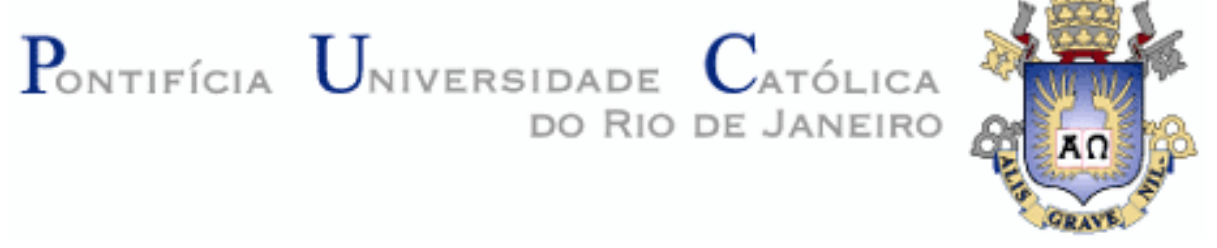

Nayara Nogueira da Costa

Design de Armazéns: uma revisão sistemática da literatura.

Dissertação de Mestrado

Dissertação apresentada como requisito parcial para obtenção do grau de Mestre pelo Programa de Pós-Graduação em Engenharia de Produção do Departamento de Engenharia Industrial da PUC-Rio.

Orientador: Prof. Nelio Domingues Pizzolato

Rio de Janeiro

Agosto de 2012 


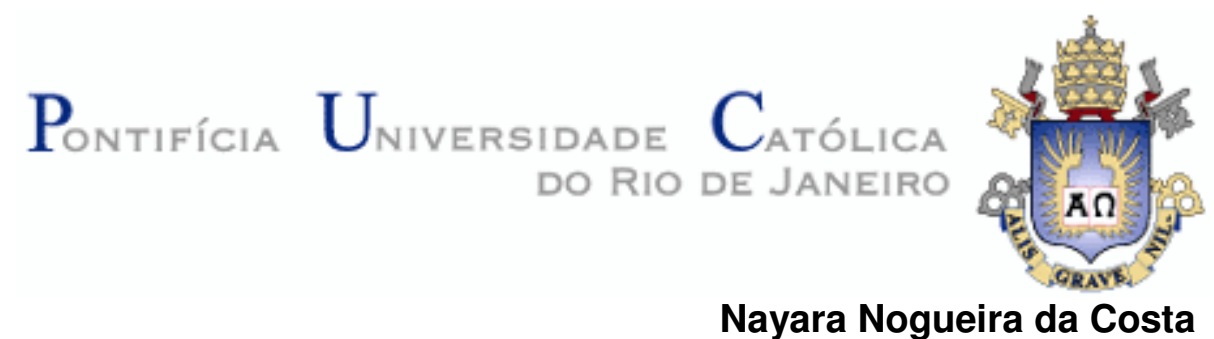

\section{Design de Armazéns: uma revisão sistemática da literatura.}

Dissertação apresentada como requisito parcial para obtenção do grau de Mestre pelo Programa de Pós-Graduação em Engenharia de Produção da PUC-Rio. Aprovada pela Comissão Examinadora abaixo assinada.

Prof. Nelio Domingues Pizzolato

Orientador

Departamento de Engenharia Industrial - PUC-Rio

Prof. Paulo Roberto Tavares Dalcol

Departamento de Engenharia Industrial - PUC-Rio

Prof. José Eugenio Leal

Departamento de Engenharia Industrial - PUC-Rio

Prof. José Eugenio Leal

Coordenador Setorial do Centro Técnico e Científico - PUC-Rio 
Todos os direitos reservados. É proibida a reprodução total ou parcial do trabalho sem autorização da universidade, do autor e do orientador.

\section{Nayara Nogueira da Costa}

Graduou-se em Engenharia de Produção pela Universidade do Estado do Pará, em Belém - PA. Durante a graduação, estagiou na empresa GV Consulting, prestando serviços em análise de processo, e na empresa Líder Supermercados e Magazine, onde participou da implantação do novo Centro de Distribuição para abastecimentos das lojas do Grupo. Depois de graduada, ingressou no Programa de Pós-graduação em Engenharia de Produção do Departamento de Engenharia Industrial da PUC-Rio para obtenção do título de Mestre.

\section{Ficha Catalográfica}

Costa, Nayara Nogueira da

Design de armazéns: uma revisão sistemática da literatura / Nayara Nogueira da Costa ; orientador: Nelio Domingues Pizzolato. - 2012.

99 f. : il. (color.) ; $30 \mathrm{~cm}$

Dissertação (mestrado)-Pontifícia Universidade Católica do Rio de Janeiro, Departamento de Engenharia Industrial, 2012.

Inclui bibliografia

1. Engenharia Industrial - Teses. 2. Design e planejamento de instalações. 3. Armazenagem. 4. Movimentação de materiais. 5. Gestão da cadeia de suprimentos. I. Pizzolato, Nelio Domingues. II. Pontifícia Universidade Católica do Rio de Janeiro. Departamento de Engenharia Industrial. III. Título. 


\section{Agradecimentos}

Primeiramente, agradeço a Deus, a quem devo tudo o que sou. Ele me deu o dom da vida e a graça de concluir esta jornada e seguir firme na caminhada pela realização profissional e pessoal.

Agradeço ao meu pai, exemplo de trabalhador e empreendedor, sempre me aconselhou nos momentos de indecisão e fez crescer em mim a vontade de vencer. Agradeço também a minha mãe, sempre um ombro amigo, sempre uma companheira. Quero agradecer também aos meus irmãos, Nathália e Nonato Segundo que sempre torceram por mim.

Ao meu Professor Nélio Pizzolato pela orientação, feedbacks sempre oportunos e por partilhar um pouco de sua vasta experiência.

Ao Professor Augusto Reis, sempre disponível a ajudar e motivar, que me ensinou muito e me fez enxergar que o que levarei de mais valioso do mestrado será a disciplina e resiliência.

À CAPES pela ajuda financeira que viabilizou grande parte desta trajetória. Aos professores e funcionários do Departamento Industrial da PUC pelo conhecimento transmitido e apoio sempre que necessário.

Gostaria de agradecer também a amiga Luciana Marçal, que me deu muita força para que pudesse concluir o mestrado, seja dando um leve puxão de orelha, seja revisando o texto ou vindo ao Rio de Janeiro para recarregar as energias. Valeu, amiga!

Aos amigos que conquistei com o mestrado, em especial gostaria de agradecer a Sara Solange, parceira nesta empreitada que foi a nossa vinda ao Rio de Janeiro, a Anaglória Lima e Ana Carla Pereira, amigas e confidentes que levarei para sempre, e ao João Victor Arruda, que neste último ano me motivou e soube entender as minhas ausências, com certeza uma grata surpresa na minha vida.

A tantos outros que me acompanharam e estiveram ao meu lado e por tudo que me ofereceram de bom. 


\section{Resumo}

da Costa, Nayara Nogueira; Pizzolato, Nélio Domingues. Design de Armazéns: uma revisão sistemática da literatura. Rio de Janeiro, 2012. 99p. Dissertação de Mestrado Departamento de Engenharia Industrial, Pontifícia Universidade Católica do Rio de Janeiro.

A implantação de instalações de armazenagem é vista como uma fonte de aumento do nível de serviço e redução de custos ao longo da cadeia de suprimentos. A presente dissertação busca analisar o estado do arte a respeito de design de armazéns utilizando a metodologia de revisão sistemática de literatura. O período de publicações levantado foi do ano de 1999 até 2011 e a base de dados eletrônica explorada foi a Science Direct. Para guiar a revisão foi elaborado um modelo conceitual contemplando as decisões que fazem parte do escopo do design de armazéns, o qual foi chamado de framework. O framework está dividido em três grandes partes. A primeira parte, input, representa as decisões que precedem ao desenho e implantação das operações de armazenagem em uma instalação e que impactam diretamente nesta fase. A segunda parte é desenho e implantação, e traz as decisões mais relevantes para instalar um armazém e quais as métricas usadas para acompanhar a performance das operações do armazém. E por fim, tem-se a última parte, output que representa o objetivo final do design de armazém para a empresa. A forma como o framework está estruturado sugere que os níveis de decisões são interativos, não podendo ser analisados separadamente, fato comprovado pela análise dos trabalhos encontrados. Por fim, pretende-se que esta dissertação sirva com uma ferramenta para profissionais de design de armazéns, bem como suporte a pesquisas futuras sobre o tema.

\section{Palavras-chave}

Design e planejamento de instalações; Armazenagem; Movimentação de materiais; Gestão da cadeia de suprimentos. 


\section{Abstract}

da Costa, Nayara Nogueira; Pizzolato, Nélio Domingues (Advisor). Warehouse Design: a systematic literature review. Rio de Janeiro, 2012. 99p. MSc. Dissertation Departamento de Engenharia Industrial, Pontifícia Universidade Católica do Rio de Janeiro.

The deployment of warehousing facilities is seen as a source of increased level of service and reducing costs along the supply chain. This master thesis aims to analyze the state of the art regarding the design of warehouses using the methodology of systematic literature review. The period of publication raised ranges from 1999 until 2011 and Science Direct was the electronic database explored. To guide the review a conceptual model was developed considering the decisions that are beyond the scope of the design of warehouses, which was called a framework. The framework is divided into three major parts. The first part, input, represents the decisions that precede the design and deployment of warehousing operations in a facility and which directly impact at this stage. The second part is the design and deployment, and brings the most important decisions to install a warehouse and what metrics are used to evaluate the performance of warehouse operations. Finally, the last part, output, representes the the final goal of designing a warehouse for the company. The way the framework is structured suggests that levels of decisions are interactive and can not be analyzed separately, a fact corroborated by the analysis of the studies found. Finally, it is expected that the present master thesis becomes a tool to warehouse design professionals, as well as support for future research on the subject.

\section{Keywords}

Facilities planning and design; Warehousing; Material Handling; Supply chain management. 


\section{Sumário}

1 INTRODUÇÃO

1.1. Apresentação do Problema 11

1.2. Objetivos 12

1.3. Estrutura da Dissertação 13

2 FRAMEWORK PARA A REVISÃO SISTEMÁTICA 15

2.1. INPUT 22

2.2. DESENHO E IMPLANTAÇÃO 24

2.3. OUTPUT 27

3 MÉTODO DE PESQUISA 31

4 APRESENTAÇÃO E ANÁLISES DOS RESULTADOS 38

4.1. OVERVIEWDAS PUBLICAÇÕES 39

4.2. INPUTS 41

4.2.1. Características do Produto $\quad 41$

4.2.2. Design da Cadeia de Suprimentos 43

4.2.2.1. Localização $\quad 46$

4.2.3. Fatores Financeiros $\quad 47$

4.2.4. Fatores Operacionais 48

4.3. DESENHO E IMPLANTAÇÃO 49

4.3.1. Layout

4.3.2. Estratégia de Operação

4.3.2.1. Recebimento e Expedição

4.3.2.2. Estocagem $\quad 54$

4.3.2.3. Separação de pedido $\quad 59$

4.3.2.4. Cross Docking $\quad 64$

4.3.3. Dimensionamento de recursos $\quad 65$

4.3.4. Tecnologia da Informação e Controle $\quad 67$

4.3.5. Indicadores de desempenho e monitoração $\quad 69$

4.4. OUTPUTS $\quad 71$

5 CONSIDERAÇÕES FINAIS

6 REFERÊNCIAS $\quad 76$

APÊNDICE 1

APÊNDICE $2 \quad 88$

$\begin{array}{ll}\text { APÊNDICE } 3 & 90\end{array}$

APÊNDICE 4

APÊNDICE 5

APÊNDICE $6 \quad 95$

$\begin{array}{ll}\text { APÊNDICE } 7 & 97\end{array}$

$\begin{array}{ll}\text { APÊNDICE } 8 & 99\end{array}$ 


\section{Lista de figuras}

Figura 1: Problemas sobre Design de Armazéns.

Figura 2: Framework proposto para revisão da literatura referente à Design de Armazéns.

Figura 3: Custos aproximando de Implantação de um Armazém. 29

Figura 4: Metodologia Adotada.

Figura 5: Volume de artigos sobre Design de Armazéns publicados por ano (1999 a 2011).

Figura 6: Cadeia de suprimentos típica.

Figura 7: Exemplos de decisões sobre o Padrão de posicionamento dos unificadores de carga na área de estocagem.

Figura 8: Atividades realizadas em um armazém.

Figura 9: Duas formas mais comuns de implementar a estocagem baseada na classificação ABC.

Figura 10: Classificação dos sistemas de separação. 62

Figura 11: Design de um sistema AS/RS. 66 


\section{Lista de tabelas}

Tabela 1: Etapas e práticas comuns da meta-análise. 32

Tabela 2: Checklist de Avaliação dos Trabalhos. 37

Tabela 3: Artigos selecionados para revisão. 38

Tabela 4: Distribuição dos artigos por periódico em publicação. 40

Tabela 5: Classificação das publicações analisadas. 41

Tabela 6: Característica do produto que influenciam nas decisões de Design de Armazéns. 41

Tabela 7: Custos considerados nos trabalhos analisados. 47

Tabela 8: Fatores operacionais considerados nos modelos. 48

Tabela 9: Lista das Publicações sobre layout e os problemas abordados. 52

Tabela 10: Modelos sobre estocagem. 58

Tabela 11: Modelos sobre separação de pedido. 63 
Quem quer passar além do Bojador Tem que passar além da dor. Deus ao mar o perigo e o abismo deu, Mas nele é que espelhou o céu. 